\title{
Molecular Phylogenomics and Population Structure of Phytophthora pluvialis
}

\author{
Javier F. Tabima,, ${ }^{1,2}$ Lilah Gonen, ${ }^{1}$ Mireia Gómez-Gallego, ${ }^{3,4,5}$ Preeti Panda,,, 6 Niklaus J. Grünwald,7 Everett M. Hansen, ${ }^{1}$ \\ Rebecca McDougal, ${ }^{3}$ Jared M. LeBoldus, ${ }^{1,8, \dagger}$ and Nari M. Williams ${ }^{3,6, \dagger}$ \\ ${ }^{1}$ Department of Botany and Plant Pathology, Oregon State University, 2082 Cordley Hall, Corvallis, OR 97331, U.S.A. \\ ${ }^{2}$ Department of Biology, Clark University, The Lasry Center for Bioscience, Worcester, MA 01610, U.S.A. \\ ${ }^{3}$ New Zealand Forest Research Institute (Scion), 49 Sala Street, Te Papa Tipu Innovation Park, Private Bag 3020, Rotorua 3046, New Zealand \\ ${ }^{4}$ Department of Forest Mycology and Plant Pathology, Swedish University of Agricultural Sciences, Box 7026, 750 07 Uppsala, Sweden \\ 5 UMR IAM-Interactions Arbres-Microorganismes, Université de Lorraine, INRAE, Nancy 54000, France \\ ${ }^{6}$ Department of Pathogen Ecology and Control, Plant and Food Research, Private Bag 1401, Havelock North 4130, New Zealand \\ ${ }^{7}$ USDA Agricultural Research Service, Horticultural Research Unit, 3420 NW Orchard Ave., Corvallis, OR 97331, U.S.A. \\ ${ }^{8}$ Department of Forest Engineering, Resources and Management, Oregon State University, Peavy Forest Science Center, Corvallis, OR \\ 97331, U.S.A. \\ Accepted for publication 6 October 2020.
}

\begin{abstract}
Phytophthora pluvialis is an oomycete that was first isolated from soil, water, and tree foliage in mixed Douglas-fir-tanoak forests of the U.S. Pacific Northwest (PNW). It was then identified as the causal agent of red needle cast of radiata pine (Pinus radiata) in New Zealand (NZ). Genotyping-bysequencing was used to obtain 1,543 single nucleotide polymorphisms across 145 P. pluvialis isolates to characterize the population structure in the PNW and NZ. We tested the hypothesis that P. pluvialis was introduced to NZ from the PNW using genetic distance measurements and population structure analyses among locations between countries. The low genetic distance, population heterozygosity, and lack of geographic structure in NZ suggest a single colonization event from the United States followed by clonal expansion in NZ. The PNW Coast Range was proposed as a presumptive center of origin of the currently known distribution of $P$. pluvialis based on its

geographic range and position as the central cluster in a minimum spanning network. The Coastal cluster of isolates were located at the root of every U.S. cluster and emerged earlier than all NZ clusters. The Coastal cluster had the highest degree of heterozygosity $(\mathrm{Hs}=0.254)$ and median pairwise genetic distance $(0.093)$ relative to any other cluster. Finally, the rapid host diversification between closely related isolates of $P$. pluvialis in NZ indicate that this pathogen has the potential to infect a broader range of hosts than is currently recognized.

Keywords: diseases in natural plant populations, Douglas-fir, ecology, epidemiology, forest pathology, genomics, invasive disease, microbegenome sequencing, oomycetes, population biology, phylogenomics, Phytophthora pluvialis, population genomics, radiata pine
\end{abstract}

The introduction of exotic plant pathogens poses risks to agroecosystems, natural environments, and human health (CorredorMoreno and Saunders 2020; Fisher et al. 2012; Scott et al. 2019; Wingfield et al. 2015). For example, the introduction of Phytophthora infestans (Montagne) de Bary to Europe from Mexico in the 19th century, caused the Irish Potato Famine. This famine was a result of the near-total failure of Ireland's primary food staple, the potato, following an outbreak of $P$. infestans. The loss of potatoes led to widespread starvation and the Irish diaspora (Goss et al. 2014). Recently, introduced plant pathogens such Phytophthora ramorum Werres, De Cock \& Man in 't Veld, the cause of sudden oak death (Cobb et al. 2012), and Cronartium ribicola J.C. Fisch, the cause of white pine blister rust (Kinloch 2003), have had devastating impacts on natural ecosystems. These and other

${ }^{\dagger}$ Corresponding authors: J. M. LeBoldus; jared.leboldus@science.oregonstate.edu, and N. M. Williams; nari.williams@plantandfood.co.nz

Funding: Support was provided by the U.S. Forest Service forest health protection evaluation and monitoring program for surveying efforts (grant 17-JV-11062765710) and by MBIE (CO4X1305) and the Forest Growers Levy Trust (administered by the New Zealand Forest Owners Association) under the "Healthy trees, Healthy future" research program at Scion (NZFRI, Ltd.).

*The $\boldsymbol{e}$-Xtra logo stands for "electronic extra" and indicates there are supplementary materials published online.

The author(s) declare no conflict of interest.

(c) 2021 The American Phytopathological Society reported introductions of plant pathogens have been linked to human-mediated movement of infected plants and infested soil (Kamvar et al. 2015a; Tabima et al. 2020; Wingfield et al. 2015). Understanding the source-sink dynamics of these introductions informs policy, prevents future introductions, and can be used to mitigate disease impacts.

Microorganisms coevolve with sympatric plant species, and can cause little to no disease in their hosts within their native range (Stukenbrock and McDonald 2008). When their geographic distribution shifts, they come into contact with naïve plant species, potentially leading to new disease outbreaks (Grünwald and Flier 2005; Stukenbrock and McDonald 2008). The source of exotic pathogens can provide important information about the genetic diversity of the microbe, its native hosts, and potential sources of resistance. For example, the establishment of the center of origin of $P$. infestans in Mexico led to the identification of wild Solanaceae spp. with coevolved resistance to the pathogen. These species have been used to identify resistance genes that have been integrated into commercial potato varieties (Grünwald and Flier 2005). In the case of forest ecosystems, Bretziella fagacearum (T. W. Bretz) J. Hunt, the causal agent of oak wilt, is another example. The current range of B. fagacearum is restricted to the United States, but population genetic analyses indicate that the genetic diversity of the species is very limited in the United States and likely a result of a single clonal introduction. It has therefore been hypothesized to have been introduced from either Mexico, Central America, or northern South America despite the lack of reports from these regions (Juzwik et al. 2008). 
A similar example of long-distance movement of a plantassociated microbe is $P$. pluvialis Reeser, Sutton, \& Hansen. This oomycete was first isolated from baited streams, soil, and canopy drip in Douglas-fir (Pseudotsuga menziesii var. menziesii [Mirbel] Franco)/tanoak (Notholithocarpus densiflorus [Hook. \& Arn.] Manos, Cannon, \& Oh) forests in southwestern Oregon (OR) (Hansen et al. 2017; Reeser et al. 2013). In 2014, P. pluvialis was confirmed as the cause of red needle cast (RNC), a disease of radiata pine (Pinus radiata [D. Don]) that was first identified on the North Island of New Zealand (NZ) in 2008 (Dick et al. 2014). Radiata pine is a tree species native to coastal California (CA) and was introduced to NZ in the 19th century for use in plantation forestry (Burdon et al. 2017). Symptoms of RNC start as discrete olivecolored lesions and black resinous bands on needles. As the disease progresses the lesions change color from yellow to red brown. Needles on affected trees eventually turn a reddish color and senesce. Premature defoliation of the lower crown and occasionally the entire tree is observed. In addition, $P$. pluvialis has been isolated from olive-colored Douglas-fir needles growing near radiata pine plantations in NZ (Gómez-Gallego et al. 2019).

On the west coast of the United States $P$. pluvialis has been isolated from scattered regions in Washington (WA), OR, and CA (Brar et al. 2018; Gómez-Gallego et al. 2019; Hansen et al. 2015). A variety of approaches have been used to collect isolates including canopy drip, symptomatic needle tissue, soil baiting, and stream baiting (GómezGallego et al. 2019; Hansen et al. 2017; Reeser et al. 2013). In NZ, collections have been made from Douglas-fir plantations across both islands (Gómez-Gallego et al. 2019). The symptoms on Douglas-fir are more cryptic than those found on radiata pine. Isolations can frequently be made from olive-colored and asymptomatic needles. Since its initial description, $P$. pluvialis has been isolated from symptomatic Douglasfir and tanoak in the United States and radiata pine, Douglas-fir, patula pine (Pinus patula Schiede ex Schltdl. \& Cham.), and Eastern white pine (Pinus strobus L.) in NZ.

The discovery of $P$. pluvialis in both OR and NZ suggests humanmediated migration across the Pacific Ocean. Like other Clade 3 Phytophthora spp., P. pluvialis disperses aerially over relatively short distances, via rain splash and wind-driven rain (Dick et al. 2014; Hansen et al. 2017). Recent efforts to identify the pathway of introduction of $P$. pluvialis used 28 single nucleotide polymorphisms (SNPs). The analysis suggested two introductions of $P$. pluvialis from OR to NZ (Brar et al. 2018). The authors hypothesized that the introduction was likely due to movement of soil, water, or asymptomatic plant material (Brar et al. 2018). Similar mechanisms of anthropogenic-mediated spread have been suggested for $P$. ramorum and other plant pathogens (Davidson et al. 2008; Grünwald et al. 2012). In order to test the hypothesis of multiple introductions, the authors suggested that increasing the number of SNP and the geographic range of the sampling would be necessary.

Building on Brar et al. (2018), the sampling in the forests of the U.S. PNW was expanded and additional samples from NZ were added. These samples were collected from various hosts across the current distribution of the pathogen. Our overarching goal was to develop a better understanding of the population dynamics of $P$. pluvialis in NZ and the United States. The specific objectives were to (i) characterize the population structure of $P$. pluvialis in the United States and NZ; (ii) study the genetic diversity of $P$. pluvialis at the state/location level within these geographic regions; and (iii) examine the interaction between genetic structure and host range. To meet these objectives, 157 isolates of $P$. pluvialis were genotyped using genotyping-by-sequencing (GBS) and a population genomics framework was used to address the objectives.

\section{MATERIALS AND METHODS}

Isolate collection. A total of 157 curated isolates of $P$. pluvialis from NZ $(n=91)$ and the United States $(n=66)$ were selected for genotyping. Selections were made in order to maximize the temporal (2008 to 2018) and geographic range of sampling (Supplementary Table S1). In NZ, samples were collected from across the North and South Island from 14 regions as defined by Crosby et al. (1976): Northland (ND; $n=5$ ), Gisborne (GB; $n=36)$, Bay of Plenty (BP; $n=11$ ), Wairarapa (WA-NZ; $n=5)$, Rangitikei (RI; $n=3$ ), Taupo (TO; $n=7$ ), Taranaki (TK; $n=1$ ), Auckland (AK; $n=1)$, Coromandel (CL; $n=2)$, Hawkes Bay (HB; $n=4)$, Nelson (NN; $n=11$ ), Wellington (WN; $n=3$ ), Waikato (WO; $n=1$ ), and Wanganui (WI; $n=1$ ). The U.S. isolates were collected from 14 regions in Brookings $(n=6)$, Cascades $(n=4)$, Central OR $(n=10)$, Del Norte Co. $(n=3)$, Florence $(n=1)$, Humboldt $(n=5)$, Little Lobster $(n=2)$, Medocino $(n=9)$, North CA $(n=4)$, Port-Orford $(n=14)$, Redwood national trail $(n=1)$, South OR $(n=1)$, South WA $(n=1)$, Washington $(n=3)$, and Wheeler $(n=1)$. These regions were spread across three states: WA $(n=5), \mathrm{OR}(n=39)$, and CA $(n=22)$. All isolates of $P$. pluvialis were grown in clarified carrot broth for 4 to 5 days in the dark at $17^{\circ} \mathrm{C}$. Mycelia were harvested, washed twice, transferred to 96-well collection tubes (Qiagen, U.S.A.) and sent to the Center for Genome Research and Biocomputing (CGRB) at Oregon State University (Corvallis, U.S.A.) for genomic DNA extraction and GBS.

GBS, read mapping, and quality filtering. To reduce the complexity of the genome, a double restriction enzyme digestion was performed with the endonucleases Pst I and Mst I (New England Biolabs, Ipswich, MA, U.S.A.). All samples were sequenced on the Illumina HiSeq 3000 platform using 150-bp single end reads following the Illumina protocol (San Diego, CA, U.S.A.). Sequenced libraries were demultiplexed using the program sabre (https://github.com/najoshi/sabre). All reads are available at the NCBI SRA repository (accession number PRJNA638875).

The reads from the sequenced isolates were aligned to the reference genome of $P$. pluvialis type strain LC-9 (Studholme et al. 2016). Alignments were made using bowtie 2, with the "-very careful" option as the sole parameter added to the program (Langmead and Salzberg 2012). In order to identify which of the genetic variants represent the ancestral state, reads from the genome of $P$. agathidicida B.S. Weir, Beever, Pennycook \& Bellgard strain NZFS 3770 were also mapped to the $P$. pluvialis reference (Studholme et al. 2016). The resulting SAM file was converted into a BAM file, sorted, and indexed using SAMTools V. 2.1.2 (Li et al. 2009). P. agathidicida is a sister species found in Phytophthora Clade 5 and is the most closely related Phytophthora species with a reference genome. This polarization approach will allow more accurate prediction of the earliest emerging populations of P. pluvialis (United States or NZ).

Genomic variants were predicted using GATK V. 4.0 (McKenna et al. 2010). Initially, each sample was independently genotyped with GATK's HaplotypeCaller and the GVCF option. All samples were pooled, and common variants identified in the CombineGVCFs program. Finally, the likelihood of each variant was estimated using the GenotypeGVCFs program for all samples. The final variant files were filtered for quality using the $v c f R$ package (Knaus and Grünwald 2017) in R (R Core Team 2019). Two variant files were created for subsequent analysis: (i) P. pluvialis and $P$. agathidicida reads (combined-species dataset); and (ii) $P$. pluvialis reads only (single-species dataset).

Variants were filtered in a three-stage process. In stage one, for the combined-species dataset variant positions missing in $P$. agathidicida were removed. In stage two, for both the combinedspecies and single-species datasets, filtering was performed to remove individual variants with a minimum read depth (DP) of $<4 \times$ and greater than the 95 th percentile of each sample DP distribution. In stage three, variants were filtered by mapping quality $(\mathrm{MQ}=44)$, minimum allele frequency (MAF < 0.05), and missing data. All variant positions with any missing data were removed and finally samples with more than $70 \%$ missing data were eliminated. The 
final single-species and combined-species files were used in all subsequent analysis.

Population differentiation of $P$. pluvialis between countries. To characterize the population dynamics between the United States and NZ and identify a presumptive center of origin, we measured the degree of genetic differentiation across $P$. pluvialis samples in a hierarchical manner. Initially, comparisons between countries were made among geographic regions within countries. To identify the earliest emerging population of $P$. pluvialis from our sampled populations, a phylogenetic tree using maximum likelihood was reconstructed in RAxML (Stamatakis et al. 2008) on both the combined-species dataset and single-species dataset. In both cases, $P$. agathidicida was the outgroup used to polarize the tree. The MULTIGAMMAI model of substitution with 1,000 bootstraps was used. The degree of genetic connectivity among populations was evaluated using a minimum spanning network (MSN) constructed in the R package poppr 2.1.5 (Kamvar et al. 2014, $2015 b$ ). Finally, population differentiation was evaluated at the different hierarchies with Nei's Gst, an Fst analog estimated in the mmod 1.13 package (Winter 2012). Population heterozygosity was estimated and statistically contrasted for each population using the Hs.test function with 499 replicates using the same package (Winter 2012). Population differentiation and population heterozygosity were measured in the single-species dataset.

Population dynamics in the United States. For the singlespecies dataset the number of genetic clusters (K) of $P$. pluvialis was estimated using the find.clusters function of the adegenet package in R (Jombart 2008). The optimal number of clusters was automatically determined by goodness of fit with Bayesian inference criterion across all values of $\mathrm{K}=2$ to 10 . A discriminant analysis of principal components (Jombart et al. 2010) was performed for each sample using the predicted genetic clusters as a population index.

For each genetic cluster, heterozygosity was calculated using the Hs function in the mmod package (Winter 2012). The distribution and median estimates of pairwise genetic distance within each population were calculated using the bitwise.distance function in poppr (Kamvar et al. 2014). The index of association $\left(\mathrm{I}_{\mathrm{A}}\right)$ was calculated for each genetic cluster to identify the degree of linkage within populations. $\mathrm{I}_{\mathrm{A}}$ was calculated with the RbarD index implemented in poppr (Kamvar et al. 2014). A total of 999 RbarD permutations were performed.

To identify the degree of genetic differentiation across the predicted genetic clusters, Nei's Gst was estimated for each pairwise combination of genetic clusters as described above (Winter 2012). To evaluate the relatedness of the predicted genetic clusters, the maximum likelihood phylogenetic reconstruction from the previous step was colored by genetic cluster in ggtree (Yu et al. 2017). Similarly, the MSN was colored by genetic cluster and geographic regions in order to observe how different geographic regions were grouped within each predicted genetic cluster. To identify if the predicted genetic clusters were grouped geographically, a map of the samples colored by genetic cluster was constructed using the ggmap package in R (Kahle and Wickham 2013).

Population structure and host specificity of $\boldsymbol{P}$. pluvialis. To test the relatedness of $P$. pluvialis samples from different hosts in NZ, the host information was superimposed on the MSN and phylogenetic reconstruction of the single species dataset. The overlay was performed by creating a stratified data frame of hosts in the genind object of the single species SNP dataset in poppr (Kamvar et al. 2014) nodes were colored by host. The ML tree was colored by host using the strata host table in ggtree (Yu et al. 2017).

A

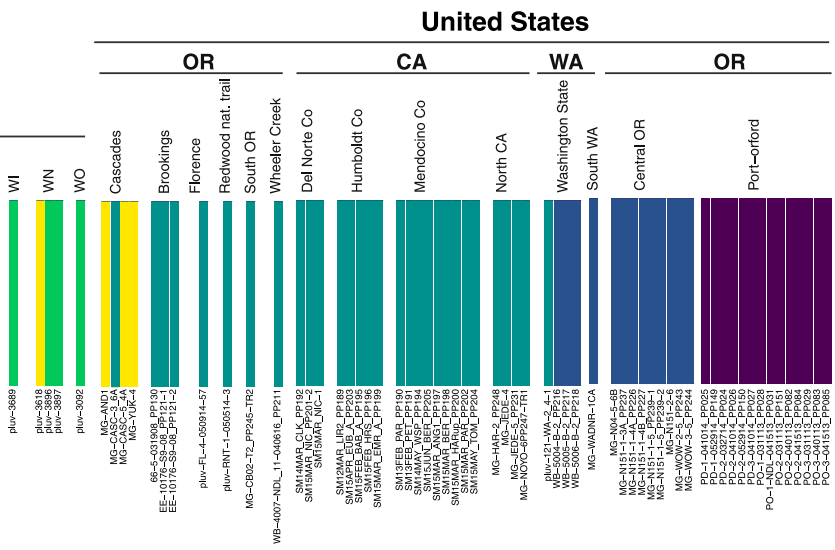

B

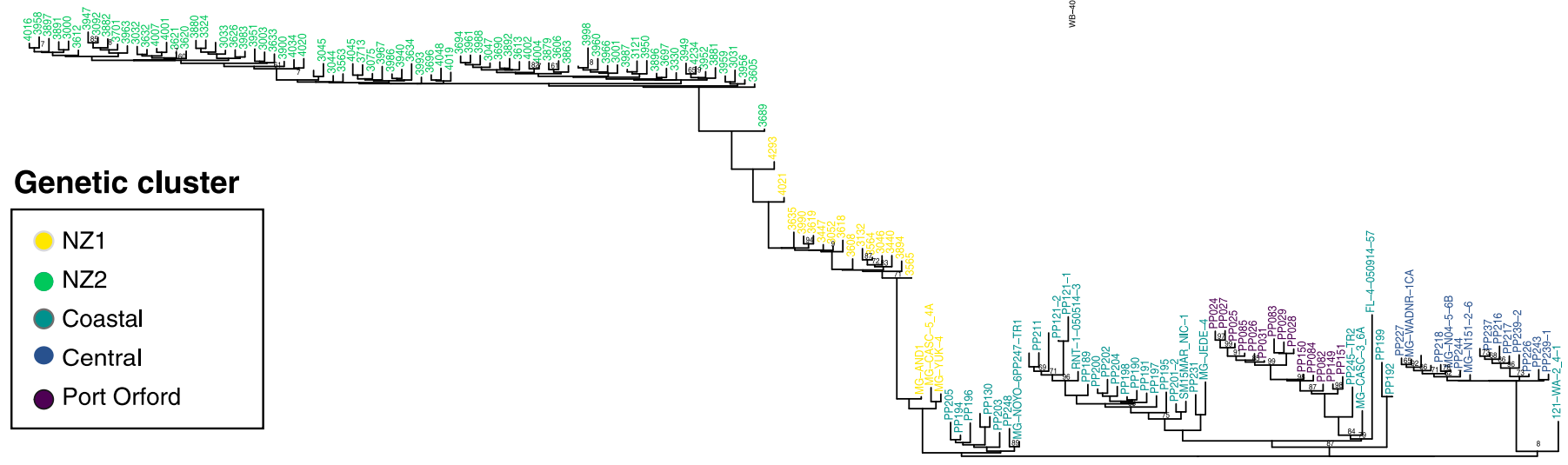

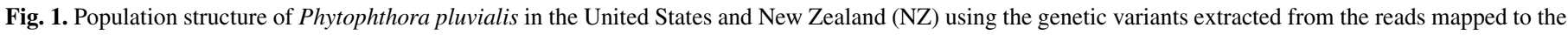

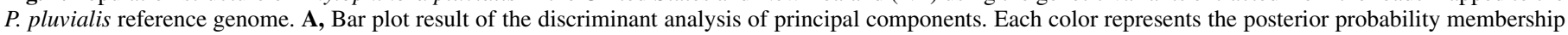

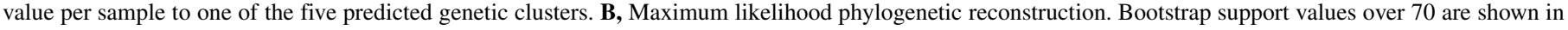

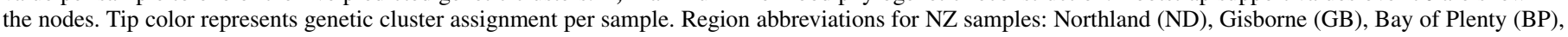

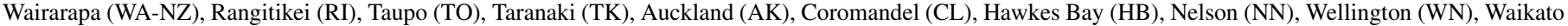
(WO), and Wanganui (WI). 


\section{RESULTS}

Summary statistics from GBS. A total of 1,543 SNPs were obtained from 145 isolates of $P$. pluvialis after variant filtering by DP, MQ, MAF, and missing data. Eight samples were removed from the single species dataset. The combined species dataset had considerably fewer variants and isolates: 156 SNPs across 78 samples after variant filtering.

Genetic differentiation between United States and NZ populations of $\boldsymbol{P}$. pluvialis. Differences between the U.S. and
NZ populations were observed across all analyses performed. The phylogenetic reconstruction with $P$. agathidicida as a root reveals that all samples from the United States are clustered in two early emerging clades, while the NZ samples were grouped in a single terminal clade (Supplementary Fig. S1A), with the exception of pluv-3635. The U.S. clades have a longer average branch length than the NZ clade, indicating a higher average number of substitutions per site. The MSN also separated the U.S. samples from the NZ samples (Supplementary Fig. S1B). Pairwise genetic distances from the MSN showed that the U.S. samples had an
A

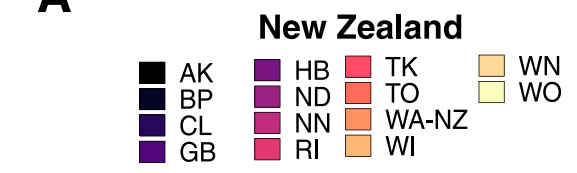

\section{Site of collection (Circles)}

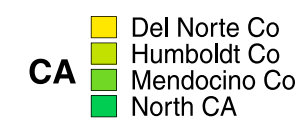
USA

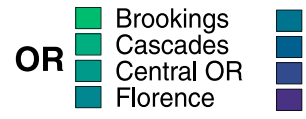

Port-orford

Redwood nat. trail WA $\square$ South WA

Wheeler Creek

WA

Washington State

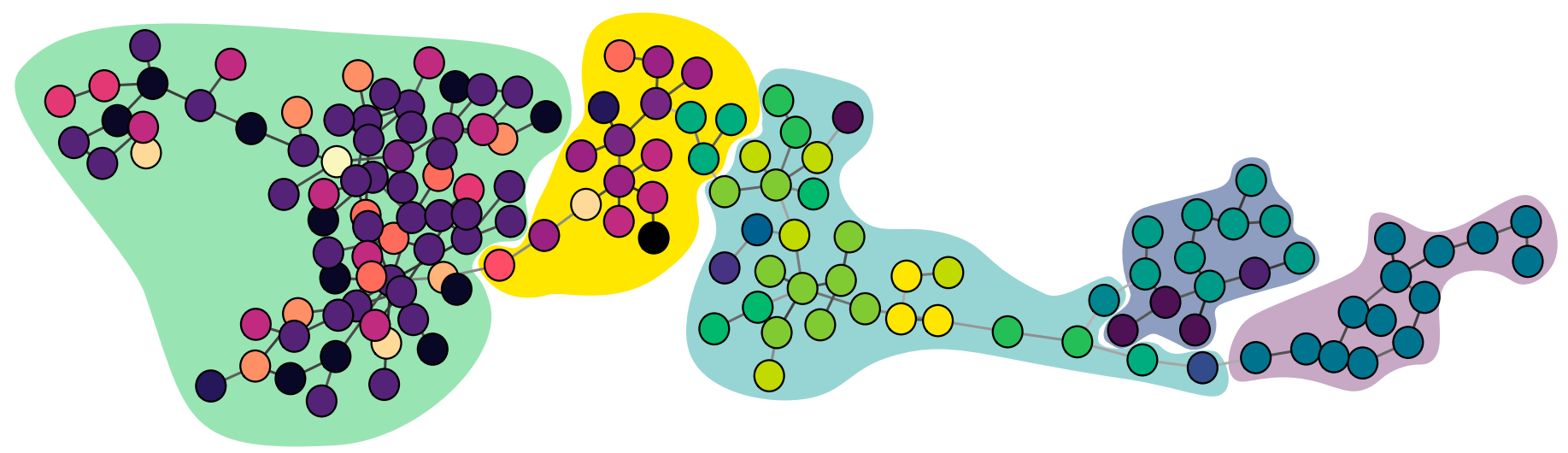

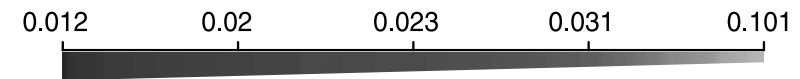

Pairwise distance

\section{Genetic cluster}

NZ2

Coastal 3 Port Orford

NZ1 Central

B

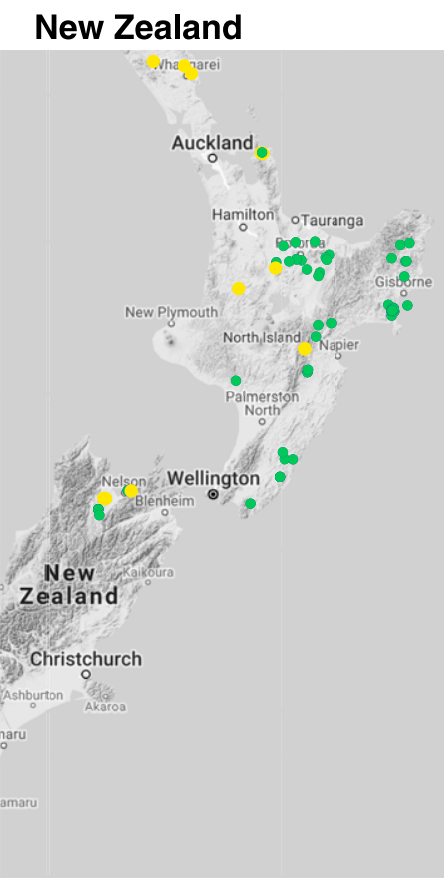

U.S.A P.N.W

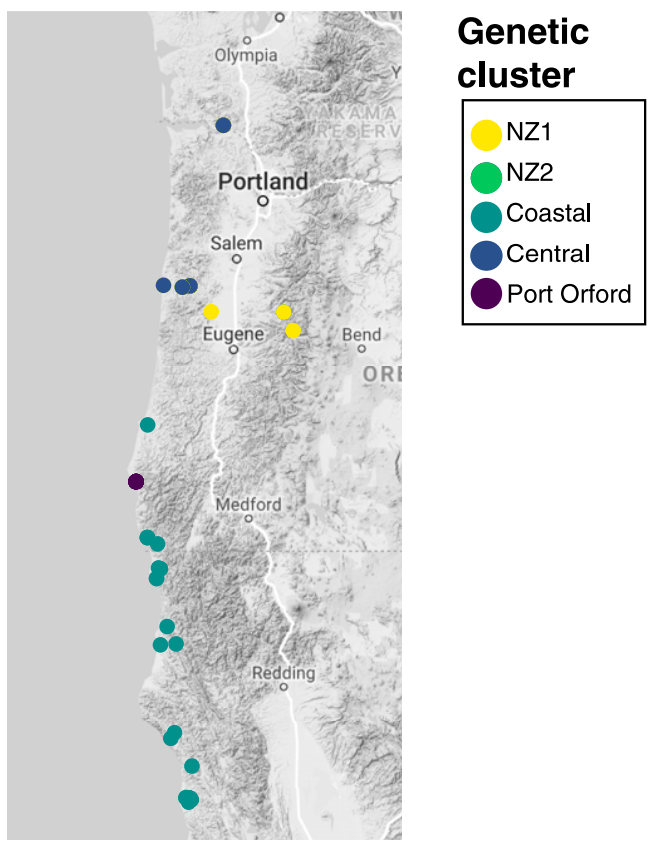

Fig. 2. Population dynamics of Phytophthora pluvialis. A, Minimum spanning network reconstructed using pairwise distances between samples. Node color represents geographic regions of each sample. Edge thickness represents genetic distance between samples. Overlaying polygons represent each predicted genetic cluster. B, Geographic distribution of samples used in this study. Colors represent sample membership to each predicted genetic cluster. Region abbreviations for New Zealand (NZ): Northland (ND), Gisborne (GB), Bay of Plenty (BP), Wairarapa (WA-NZ), Rangitikei (RI), Taupo (TO), Taranaki (TK), Auckland (AK), Coromandel (CL), Hawkes Bay (HB), Nelson (NN), Wellington (WN), Waikato (WO), and Wanganui (WI). 
overall greater genetic distance among samples than those collected in NZ. Population heterozygosity metrics indicated significantly higher heterozygosity in the United States $(0.285)$ compared with NZ (0.239) (Hs.test, $P=0.002$ ). The NZ sample pluv-3635 is clustered in a different clade than the other NZ samples. It is more closely related to MG-AND1 and MG-YUK-4, two samples from the Cascades cluster in OR. This placement is consistent in both the phylogenetic reconstruction and the MSN.

Genetic differentiation among regions in the United States and NZ. Stratification of isolates by region within countries revealed low pairwise differences in genetic diversity between regions (Supplementary Figs. S3 and S4). In most cases, the closer the regions the less genetic differentiation. However, there were several exceptions (Supplementary Figs. S2 and S3). Across the United States, the Central OR, South WA, Florence OR, and WA state regions were all similar (Gst < 0.05) compared with the other geographic regions in the United States. Additionally, samples from the Port-Orford region had the highest genetic differentiation (Gst > 0.12) when compared with all U.S. regions, with the exception of South OR and Florence OR. In the case of NZ, the only geographic area with strong evidence for genetic differentiation was ND. Pairwise comparisons of genetic differentiation between ND and GB, BP, WA-NZ, RI, and TO regions had an Gst > 0.12 (Crosby et al. 1976). The pairwise genetic differentiation indicates low differentiation between regions within countries. Two regions from NZ, TK and WI, were weakly differentiated (Gst $<0.1$ ) when compared with all the U.S. regions with the exception of Central OR and Port-Orford.

Population dynamics of $P$. pluvialis between its U.S. center of origin and NZ. Five genetic clusters were identified across the United States $(n=3)$ and NZ $(n=2)$ (Fig. 1A and 2A and B). The Coastal, Central, and Port-Orford genetic clusters grouped most U.S. samples. The remaining samples were from the Cascades region, and clustered within NZ1. The Coastal cluster grouped all southern OR and northern CA coastal samples. The Central cluster grouped all northern OR and WA samples. Port-Orford samples grouped in their own cluster with a single sample from the Cascades. NZ1 grouped all samples from the regions of ND, AK, and $\mathrm{TK}$, three from NN, two from $\mathrm{HB}$, one from $\mathrm{WN}$ in NZ, and three samples from the OR Cascades region in the United States. NZ2 was exclusive to NZ and grouped all samples from GB, BP, WA, and RI, four samples from TO, eight from NN, two from HB, two from WN, and one from CL.

The Coastal genetic cluster is the most diverse of the genetic clusters, both in terms of heterozygosity and pairwise genetic distance, while the Port-Orford and NZ2 clusters are the least diverse (Table 1, Supplementary Fig. S4). The Coastal genetic cluster had the lowest genetic differentiation when compared with any other genetic cluster (Gst $<0.15$, Table 2 ). All other pairwise comparisons had similar levels of genetic differentiation (Gst $>$ 0.18). The $I_{A}$ simulations indicate that the NZ2 and Port-Orford clusters have the largest simulated values of RbarD compared with the other clusters. This indicates a higher degree of genetic linkage within these populations and a higher degree of clonal reproduction compared with the other populations (Supplementary Fig. S5).

The Coastal genetic cluster had more connections to other genetic clusters in the MSN. Phylogenetic reconstruction revealed that the Coastal cluster was always positioned with the earliest emerging clade, suggesting the Coastal cluster is the center of origin of P. pluvialis. All population clustering metrics indicated that MGYUK-4, MG-CASC-5, and MG-AND-1, isolated from Douglas-fir in the Cascades region of $\mathrm{OR}$, are the most closely related to the NZ samples clustered within NZ1.

Genetic structure and host associations in NZ P. pluvialis samples. Host overlay on the MSN indicates no correlation between the genetic cluster, phylogenetic clade, or geographic region of the isolate with host specificity (Fig. 3). All samples from the United States were exclusively isolated from Douglas-fir and as a result not included in this analysis. The phylogenetic tree indicates no host specificity or directional selection to date.

\section{DISCUSSION}

The hypothesis of a single colonization of $P$. pluvialis giving rise to the NZ RNC epidemic is supported by the analysis and results described above. A demographic analysis of the 147 isolates indicates that unidirectional migration from the United States to NZ is the most parsimonious explanation for the observed genetic patterns (Supplementary Fig. S2). The source of this colonization event was likely the Cascades region in OR. Weak genetic differentiation among isolates from the Cascades region in the United States and the Northland and Nelson regions in NZ resulted in their grouping into a single genetic cluster. In addition, all measures of genetic diversity from U.S. samples were greater than the samples from NZ, regardless of the metric used for comparison (Supplementary Fig. S3).

The increased number of molecular markers, as a result of the GBS, improved the characterization of the U.S. and NZ source-sink dynamics. This can best be seen when comparing the single-species versus combined-species datasets. The analysis with the combinedspecies dataset groups one NZ isolate within the U.S. samples (Supplementary Fig. S1A). In this reduced dataset the sample labeled pluv-3635 is more closely related to the U.S. samples than to $\mathrm{NZ}$ samples. When the same analysis is conducted with the larger single-species dataset (1,543 SNPs) this isolate is repositioned within the NZ1 genetic cluster. The likely explanation for this change is the small number of molecular markers retained $(n=156)$ when using the distantly related $P$. agathidicida to polarize the ML tree (Supplementary Fig. S1A). Furthermore, a similar pattern was noted by Brar et al. (2018) who suggested that the NZ outbreak was a result of two separate introductions. Their analysis used 27 SNPs rather than the 1,543 SNPs identified in the single-species dataset described herein. The combination of the number of markers in this analysis and the population genetics metrics (Figs. 1B and 2A and B) strongly support the hypothesis of a single introduction of $P$. pluvialis into NZ.

TABLE 2. Population differentiation via Nei's Gst for all pairwise comparisons of genetic clusters predicted for Phytophthora pluvialis

\begin{tabular}{lcccc}
\hline Genetic cluster & Coastal & NZ2 & Central & Port-Orford \\
\hline NZ2 & 0.144 & - & - & - \\
Central & 0.155 & 0.258 & - & - \\
Port-Orford & 0.086 & 0.223 & 0.235 & - \\
NZ1 & 0.153 & 0.184 & 0.231 & 0.230 \\
\hline
\end{tabular}

TABLE 1. Summary statistic for genetic diversity and linkage measurements for Phytophthora pluvialis genetic clusters

\begin{tabular}{|c|c|c|c|}
\hline Genetic cluster & Heterozygosity & Median pairwise genetic distance & Index of association (RbarD) \\
\hline Coastal & 0.254 & 0.093 & 0.037 \\
\hline Central & 0.228 & 0.054 & 0.034 \\
\hline Port-Orford & 0.201 & 0.032 & 0.047 \\
\hline NZ1 & 0.213 & 0.044 & 0.032 \\
\hline $\mathrm{NZ2}$ & 0.207 & 0.040 & 0.135 \\
\hline
\end{tabular}


The pattern of introduction followed by clonal expansion observed between the United States and NZ can be seen at a smaller spatial scale within the United States. The samples from the United States have high levels of genetic association over small geographic distances. This pattern resembles small, local epidemic events following initial migration of a small number of individuals into a new region. A good example of this is the regional outbreak of P. pluvialis reported for the Coast Range of OR in 2015 (Hansen et al. 2017). The Central genetic cluster appears to have originated from the Coastal genetic cluster followed by clonal expansion within the central region. This hypothesis is supported by the low genetic diversity, high population differentiation, and strong population structure found in the Central genetic cluster (Table 1). The Port-Orford genetic cluster exhibits similar characteristics. This cluster appears to have emerged in a manner similar to what was described above, movement from the Coastal

\section{A}

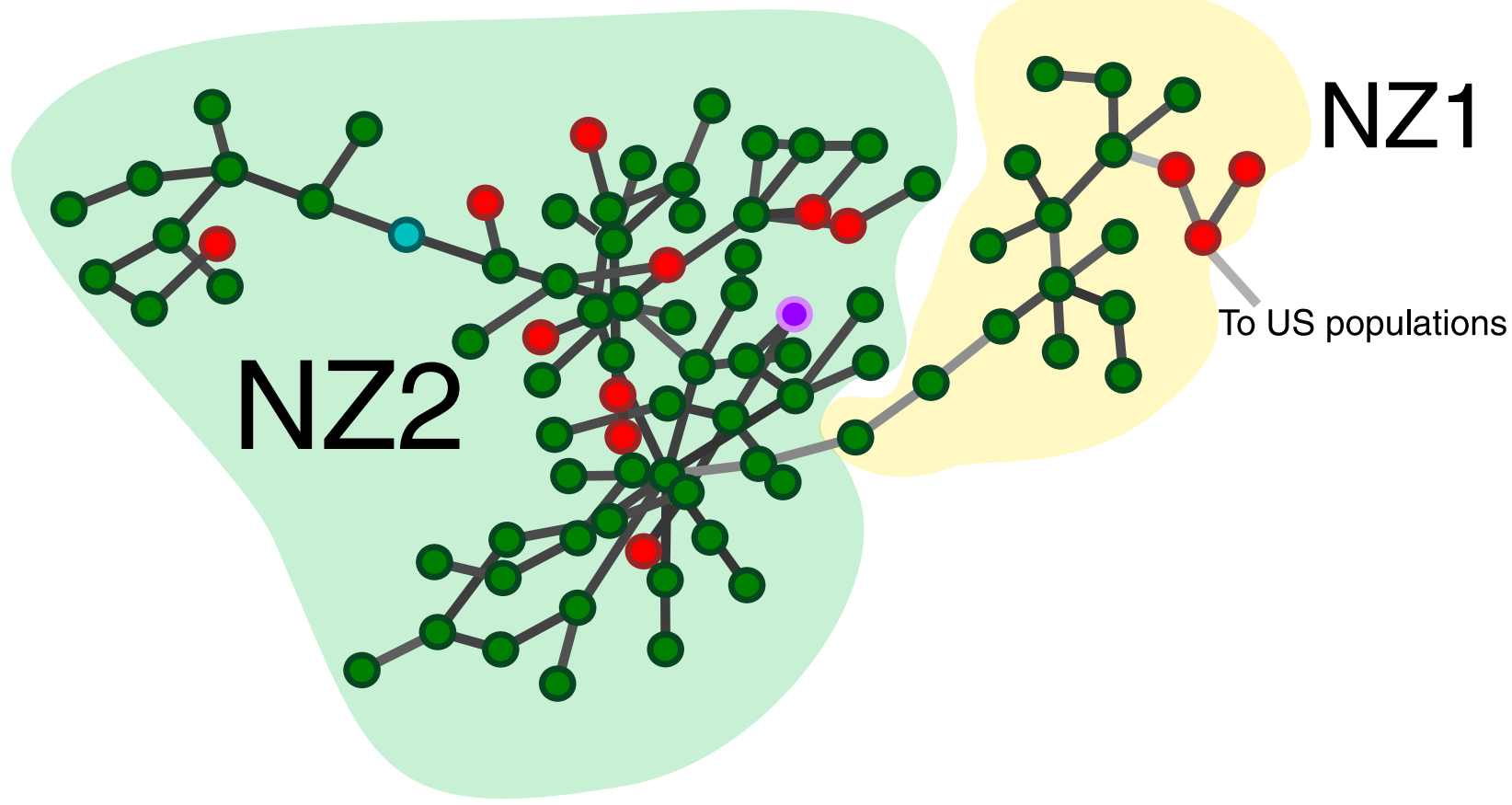

B

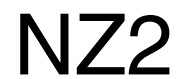

NZ1

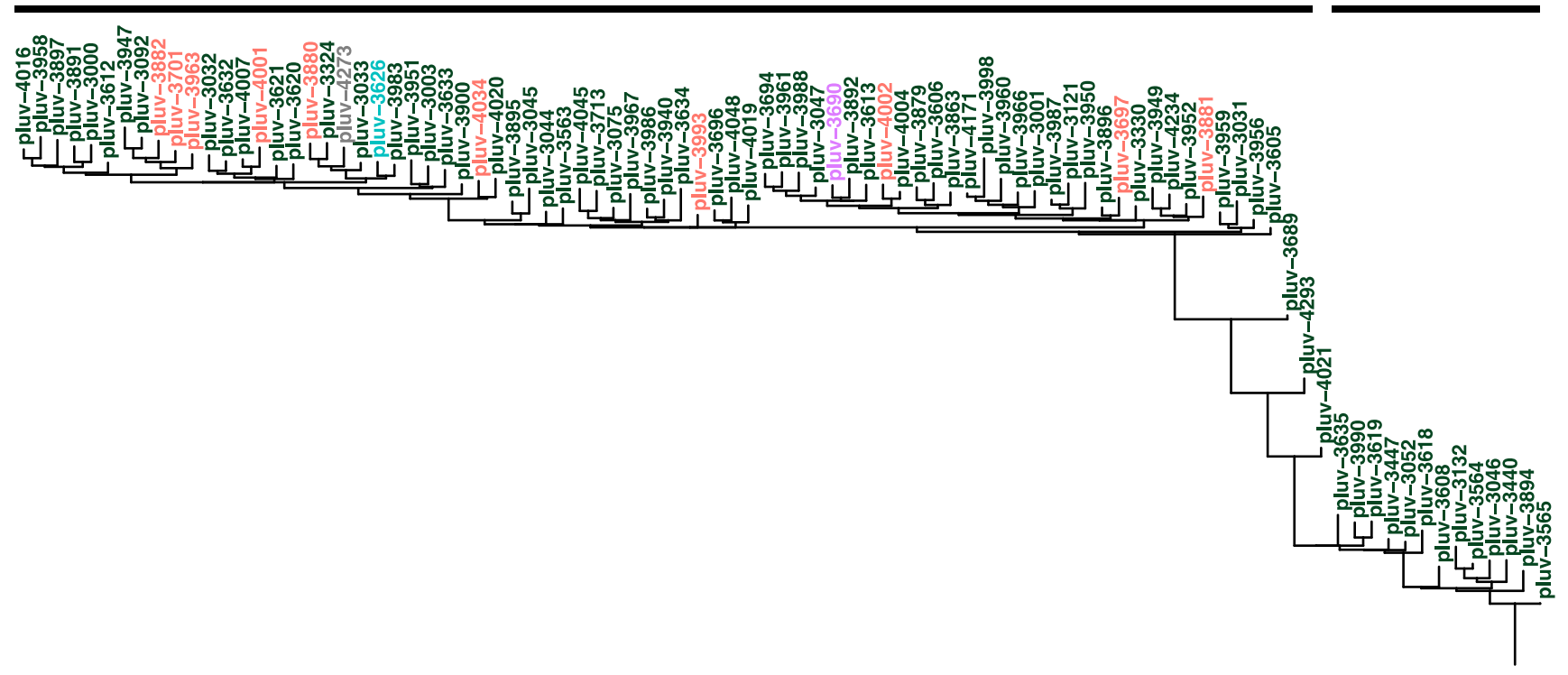

To US

\section{Associated host}

Fig. 3. Host shifts in New Zealand (NZ) samples of Phytophthora pluvialis. Each color represents the host where each sample was isolated from. A, Subset of the minimum spanning network reconstructed in Figure 2 representing samples from NZ. B, Subset of the phylogenetic reconstruction in Figure 1 representing samples from NZ. 
cluster followed by local clonal expansion (Figs. 1 and 2). Both of these examples, occurring at different times (Port-Orford in 2013 to 2014 and Central in 2017) suggest that local environmental conditions may be driving clonal expansion and outbreak development. Similar scenarios have been proposed for local dispersal of the invasive oomycete $P$. ramorum in the western United States (Peterson et al. 2014). Continued sampling both within and beyond the regions sampled in this study, and implementation of a demographic analysis would be required to detect the putative origin of $P$. pluvialis.

The question remains as to how and when P. pluvialis was introduced to NZ. The ubiquitous presence of $P$. pluvialis in the Coast Range of OR suggests many possible modes of introduction from Douglas-fir needles to infested soil. Increased biosecurity restrictions in NZ since the late 1990s and early 2000s have significantly curtailed the risk of pathogen movement from overseas, suggesting an introduction which occurred prior to these restrictions. However, once established in NZ, few measures are in place to minimize the spread of pathogens among forested areas (Froud 2018; Hood 2019).

Reports of $P$. pluvialis in the United States are limited to the forests of the PNW (Brar et al. 2018; Gómez-Gallego et al. 2020; Hansen et al. 2015). All of the U.S. samples were collected from a single conifer species, Douglas-fir; whereas the isolates collected in $\mathrm{NZ}$ came from four different conifer species. In NZ, isolates were collected from Douglas-fir $(n=9)$, radiata pine $(n=79)$, Eastern white pine $(n=1)$, and patula pine $(n=1)$ (Supplementary Table S1). However, there was no correlation between genetic or geographic cluster and host specificity. Although the isolates predominantly came from the most abundant host, Pinus radiata, multiple shifts between Douglas-fir and radiata pine were observed within the same genetic cluster and phylogenetic clade (Fig. 3) suggesting a broader host range than originally described (Hansen et al. 2017). The historic range of radiata pine spans from the northern San Francisco Bay Area in CA to northern Baja in Mexico (Millar 1999). This overlaps with the southern range of Douglas-fir. However, $P$. pluvialis has not been recovered from radiata pine in $\mathrm{CA}$ as either a pathogen or endophyte. Although RNC has never been reported in radiata pine's native range. Further sampling efforts to determine if $P$. pluvialis can be found in alternate coniferous hosts throughout North America is warranted. Finally, it is also important to note that $P$. pluvialis is less noticeable when infecting Douglas-fir compared with radiata pine where it causes RNC. However, infected Douglas-fir needles are cast prematurely and severe defoliation in the lower canopy has been noted on occasion in both the United States and NZ (Hansen et al. 2015). This may suggest a coevolutionary history between the two species whereby $P$. pluvialis plays a role in the suppression of understory species, a pattern that is characteristic of Douglas-fir stands (Gómez-Gallego et al. 2020). This potential coevolution and mutualistic ecological interactions between both species requires further exploration.

The population structure approach presented here suggests that P. pluvialis could have originated in the coastal Douglas-fir forests of the PNW where the population exhibits some characteristics of a center of origin. From this region, $P$. pluvialis was introduced at least once to NZ. Following the introduction, the pathogen was able to infect a wider range of host species than described in the PNW. These rapid host shifts may have been a contributing factor in its establishment and impact on the exotic radiata pine plantations grown throughout temperate regions of NZ. However, more evidence should be presented to support this hypothesis. The recent discovery of $P$. pluvialis has not permitted extensive sampling in areas of North America and NZ. As a result, the potential exists for undiscovered populations of $P$. pluvialis outside of these locations. In order to establish where $P$. pluvialis may have originated, more extensive sampling over a wide geographic area is required. This may reveal both the center of origin of this pathogen and other possible routes of migration.
Future work should focus on increasing sample size, in terms of both geographic area and hosts sampled, in North America and NZ. A focus on whole genome resequencing with a higher depth of coverage would further increase the number of alleles that can be detected and provide a clearer view of the genetic diversity of this pathogen. Finally, demographic modeling of migration scenarios and history to test different routes of introduction and migration would be valuable. This work highlights the importance of phylogenomics to identify sources of forest pathogens that can potentially endanger naïe hosts.

\section{ACKNOWLEDGMENTS}

We thank Kristen Chadwick, Holly Kearns, and Ellen Goheen with the USFS for help with isolate collections; Sarah Navarro, Alan Kanaskie, and Danny Nordlander with the Oregon Department of Forestry for completing the U.S. collections; Rita Tetenburg and Sara Cary for help with cultures from the NZFS culture collection at Scion (NZFRI, Ltd.); and Paul Reeser and Wendy Sutton in the Hansen Lab at Oregon State University for isolating P. pluvialis.

\section{LITERATURE CITED}

Brar, S., Tabima, J. F., McDougal, R. L., Dupont, P. Y., Feau, N., Hamelin, R. C., Panda, P., LeBoldus, J. M., Grünwald, N. J., Hansen, E. M., and Bradshaw, R. E. 2018. Genetic diversity of Phytophthora pluvialis, a pathogen of conifers, in New Zealand and the west coast of the United States of America. Plant Pathol. 67:1131-1139.

Burdon, R. D., Li, Y., Suontama, M., and Dungey, H. S. 2017. Genotype $\times$ site $\times$ silviculture interactions in radiata pine: Knowledge, working hypotheses and pointers for research. N. Z. J. For. Sci. 47:6.

Cobb, R. C., Filipe, J. A. N., Meentemeyer, R. K., Gilligan, C. A., and Rizzo, D. M. 2012. Ecosystem transformation by emerging infectious disease: Loss of large tanoak from California forests. J. Ecol. 100:712-722.

Corredor-Moreno, P., and Saunders, D. G. 2020. Expecting the unexpected: Factors influencing the emergence of fungal and oomycete plant pathogens. New Phytol. 225:118-125.

Crosby, T. K., Dugdale, J. S., and Watt, J. C. 1976. Recording specimen localities in New Zealand: An arbitrary system of areas and codes defined. N. Z. J. Zool. 3:69.

Davidson, J. M., Patterson, H. A., and Rizzo, D. M. 2008. Sources of inoculum for Phytophthora ramorum in a redwood forest. Phytopathology 98: 860-866.

Dick, M. A., Williams, N. M., Bader, M. K. F., Gardner, J. F., and Bulman, L. S. 2014. Pathogenicity of Phytophthora pluvialis to Pinus radiata and its relation with red needle cast disease in New Zealand. N. Z. J. For. Sci. 44:6.

Fisher, M. C., Henk, D. A., Briggs, C. J., Brownstein, J. S., Madoff, L. C., McCraw, S. L., and Gurr, S. J. 2012. Emerging fungal threats to animal, plant and ecosystem health. Nature 484:186-194.

Froud, K. J. 2018. Forest Biosecurity Committee Review 2018 Operational Biosecurity: Review of the biosecurity preparedness of the forestry industry to identify opportunities to improve biosecurity outcomes for forestry. A report prepared for the NZ Forest Owners Association by Biosecurity Research Limited.

Gómez-Gallego, M., LeBoldus, J. M., Martin Karl-Friedrich Bader, M. K.-F., Hansen, E. M., Donaldson, L., and Williams, N. M. 2019. Contrasting the pathogen loads in co-existing populations of Phytophthora pluvialis and Nothophaeocryptopus gaeumannii in Douglas fir plantations in New Zealand and the Pacific Northwest United States. Phytopathology 109: 1908-1921.

Gómez-Gallego, M., Williams, N., Leuzinger, S., Scott, P. M., and Bader, M. K. F. 2020. No carbon limitation after lower crown loss in Pinus radiata. Ann. Bot. (Lond.) 125:955-967.

Goss, E. M., Tabima, J. F., Cooke, D. E., Restrepo, S., Fry, W. E., Forbes, G. A., Fieland, V. J., Cardenas, M., and Grünwald, N. J. 2014. The Irish potato famine pathogen Phytophthora infestans originated in central Mexico rather than the Andes. Proc. Natl. Acad. Sci. 111:8791-8796.

Grünwald, N. J., and Flier, W. G. 2005. The biology of Phytophthora infestans at its center of origin. Annu. Rev. Phytopathol. 43:171-190.

Grünwald, N. J., Garbelotto, M., Goss, E. M., Heungens, K., and Prospero, S. 2012. Emergence of the sudden oak death pathogen Phytophthora ramorum. Trends Microbiol. 20:131-138.

Hansen, E. M., Reeser, P. W., and Sutton, W. 2017. Ecology and pathology of Phytophthora ITS clade 3 species in forests in western Oregon, USA. Mycologia 109:100-114. 
Hansen, E. M., Reeser, P. W., Sutton, W., Gardner, J., and Williams, N. 2015. First report of Phytophthora pluvialis causing needle loss and shoot dieback on Douglas-fir in Oregon and New Zealand. Plant Dis. 99:727.

Hood, I. A. 2019. How biosecure are we? Case studies with unwanted forestry pathogens and pests. A further look at New Zealand's import health standards. Report 61533. New Zealand Forest Research Institute Limited.

Jombart, T. 2008. adegenet: An R package for the multivariate analysis of genetic markers. Bioinformatics 24:1403-1405.

Jombart, T., Devillard, S., and Balloux, F. 2010. Discriminant analysis of principal components: A new method for the analysis of genetically structured populations. BMC Genet. 11:94.

Juzwik, J., Harrington, T. C., MacDonald, W. L., and Appel, D. N. 2008. The origin of Ceratocystis fagacearum, the oak wilt fungus. Annu. Rev. Phytopathol. 46:13-26.

Kahle, D., and Wickham, H. 2013. ggmap: Spatial visualization with ggplot2. R J. 5:144-161.

Kamvar, Z. N., Brooks, J. C., and Grünwald, N. J. 2015a. Novel R tools for analysis of genome-wide population genetic data with emphasis on clonality. Front. Genet. 6:208.

Kamvar, Z. N., Larsen, M. M., Kanaskie, A. M., Hansen, E. M., and Grünwald, N. J. 2015b. Spatial and temporal analysis of populations of the sudden oak death pathogen in Oregon forests. Phytopathology 105:982-989.

Kamvar, Z. N., Tabima, J. F., and Grünwald, N. J. 2014. Poppr: An R package for genetic analysis of populations with clonal, partially clonal, and/or sexual reproduction. PeerJ 2:e281.

Kinloch, B. B., Jr. 2003. White pine blister rust in North America: Past and prognosis. Phytopathol 93:1044-1047.

Knaus, B. J., and Grünwald, N. J. 2017. vefr: A package to manipulate and visualize variant call format data in R. Mol. Ecol. Resour. 17:44-53.

Langmead, B., and Salzberg, S. L. 2012. Fast gapped-read alignment with Bowtie 2. Nat. Methods 9:357-359.

Li, H., Handsaker, B., Wysoker, A., Fennell, T., Ruan, J., Homer, N., Marth, G., Abecasis, G., and Durbin, R. 2009. The sequence alignment/map format and SAMtools. Bioinformatics 25:2078-2079.
McKenna, A., Hanna, M., Banks, E., Sivachenko, A., Cibulskis, K., Kernytsky, A., Garimella, K., Altshuler, D., Gabriel, S., Daly, M., and DePristo, M. A. 2010. The Genome Analysis Toolkit: A MapReduce framework for analyzing next-generation DNA sequencing data. Genome Res. 20:1297-1303.

Millar, C. I. 1999. Evolution and biogeography of Pinus radiata, with a proposed revision of its Quaternary history. N. Z. J. For. Sci. 29:335-365.

Peterson, E., Hansen, E., and Kanaskie, A. 2014. Spatial relationship between Phytophthora ramorum and roads or streams in Oregon tanoak forests. For. Ecol. Manage. 312:216-224.

R Core Team. 2019. R: A Language and Environment for Statistical Computing. R Foundation for Statistical Computing, Vienna, Austria.

Reeser, P., Sutton, W., and Hansen, E. 2013. Phytophthora pluvialis, a new species from mixed tanoak-Douglas-fir forests of western Oregon, USA. N. Am. Fungi 8:1-8.

Scott, P., Bader, M. K. F., Burgess, T., Hardy, G., and Williams, N. 2019. Global biogeography and invasion risk of the plant pathogen genus Phytophthora. Environ. Sci. Policy 101:175-182.

Stamatakis, A., Hoover, P., and Rougemont, J. 2008. A rapid bootstrap algorithm for the RAxML web servers. Syst. Biol. 57:758-771.

Studholme, D. J., McDougal, R. L., Sambles, C., Hansen, E., Hardy, G., Grant, M., Ganley, R. J., and Williams, N. M. 2016. Genome sequences of six Phytophthora species associated with forests in New Zealand. Genom. Data 7:54-56.

Stukenbrock, E. H., and McDonald, B. A. 2008. The origins of plant pathogens in agro-ecosystems. Annu. Rev. Phytopathol. 46:75-100.

Tabima, J. F., Sondreli, K., Kerio, S., Feau, N., Sakalidis, M. L., Hamelin, R., and Leboldus, J. M. 2020. Population genomic analyses reveal connectivity via human-mediated transport across Populus plantations in North America and an undescribed sub-population of Sphaerulina musiva. MPMI 33:189-199.

Wingfield, M. J., Brockerhoff, E. G., Wingfield, B. D., and Slippers, B. 2015. Planted forest health: The need for a global strategy. Science 349:832-836.

Winter, D. J. 2012. MMOD: An R library for the calculation of population differentiation statistics. Mol. Ecol. Resour. 12:1158-1160.

Yu, G., Smith, D. K., Zhu, H., Guan, Y., and Lam, T. T. Y. 2017. ggtree: An R package for visualization and annotation of phylogenetic trees with their covariates and other associated data. Methods Ecol. Evol. 8:28-36. 